hep-th/0612057

Preprint typeset in JHEP style - HYPER VERSION

SU-ITP-2006-34

SLAC-PUB-12251

December 7, 2006

\title{
Issues in Type IIA Uplifting
}

\author{
Renata Kallosh ${ }^{a *}$ and Masoud Soroush ${ }^{a, b \dagger}$ \\ ${ }^{a}$ Department of Physics, Stanford University, Stanford, CA 94305, USA \\ ${ }^{b}$ SLAC, Stanford University, Stanford, CA 94309, USA
}

\begin{abstract}
Moduli stabilization in the type IIA massive string theory so far was achieved only in the AdS vacua. The uplifting to dS vacua has not been performed as yet: neither the analogs of type IIB anti-D3 brane at the tip of the conifold, nor the appropriate D-terms have been identified. The hope was recently expressed that the F-term uplifting may work. We investigate this possibility in the context of a simplified version of the type IIA model developed in hep-th/0505160 and find that the F-term does not uplift the AdS vacua to $\mathrm{dS}$ vacua with positive $\mathrm{CC}$. Thus it remains a challenging task to find phenomenologically acceptable vacua in the type IIA string theory.
\end{abstract}

\footnotetext{
* Email: kallosh@stanford.edu

$\dagger$ Email: soroush@stanford.edu
} 


\section{Contents}

\section{Introduction}

Models with all moduli stabilized in the massive type IIA string theory are relatively simple: they engage all possible fluxes, no non-perturbative effects from the perspective of the massive ten-dimensional type IIA supergravity are required [1, 2]. A model of particular interest which we will study here, is the type IIA compactification on the $T^{6} / \mathbb{Z}_{3}^{2}$ orientifold studied in [2]. It solves the equations of motion of the 10D type IIA massive supergravity with account of local O6 and D6 sources and all possible fluxes. An interesting feature of this model is that the stabilized moduli depend on a particular integer $N$ related to certain fluxes. $N$ can be arbitrarily large, in contrast to other known models with stabilized moduli where fluxes are bounded, see for example a review on this in [3]. The action for this model, as well as for more general models in this class, was constructed in [4]. More recently, various stringy aspects of this model were studied in [5].

The uplifting to $\mathrm{dS}$ vacua for these models have not been performed so far; neither the analogs of the type IIB anti-D3 brane at the tip of the conifold nor the appropriate D-terms have been identified. The recently improved version of the Kähler uplifting [7] was not yet applied to the model in [2], so we have to wait before we know if it works.

On the other hand, recently there was a significant development with the F-term uplifting in combining the KKLT model [6] with models which separately have non-supersymmetric vacua with positive energy [8, 9, 10]. The fields in these models are expected to originate from the open string sector in intersecting brane models, however, the details still have to be worked out. If the uplifting model has small vev's comparative to Planckian scales, the leading part of the F-term uplifting does not depend on the details of the added model. Perhaps the simplest of these models is the O'KKLT model [10] combining the O'Raifeartaigh model [11] with the Coleman-Weinberg corrections and the KKLT model.

The generic feature of F-term uplifting models is that

$$
K=K_{A}+K_{B}, \quad W=W_{A}+W_{B} .
$$

Here the model $A$ is gravitational and the model $B$ is close to global susy: all dimensionful quantities in model $B$ are much smaller than the Planckian scale. In such case

$$
V_{\text {total }} \approx V_{A}+e^{K_{A}} V_{B}+\ldots
$$

The examples were proposed in [9, 10]. In string theory setting the fields in $A$ originate from the closed string sector and the fields in $B$ originate from the open string sector. 
It was suggested in [9] that the new models of F-term uplifting can be applied in type IIA models of moduli stabilization [1, 2]. Here we will study this problem for the model of the massive type IIA string theory with all moduli stabilized in [2]. There are 13 complex scalars in this model, therefore the potential is extremely complicated. 3 untwisted moduli $t_{i}, i=1,2,3$ represent the volume-axion for each of the 2 -tori $T^{6}=T^{2} \times T^{2} \times T^{2}$. There are 9 blow-up moduli, $t_{B_{A}}, A=1, \ldots, 9$, associated with 9 singular orbifold points. Finally, there is a complex dilaton-axion field $N_{0}$, coming from the quaternionic part of the geometry, only half of quaternion components, forming a complex field, remains after the orientifolding.

We will consider a somewhat simplified version of this model, in which the choice of fluxes is such that i) the stabilized axions are equal to zero, $\operatorname{Ret}_{\mathrm{i}}=0, \operatorname{Re}_{\mathrm{B}_{\mathrm{A}}}=0$ and ii) the untwisted volumes are significantly larger than blow-ups $\operatorname{Im} t_{i} \gg \operatorname{Im}_{B_{A}}$. In the regime of the validity of the supergravity approximation, this simplified model captures the features of the most general class of models. Therefore it is plausible that the situation with uplifting in this simplified model will also tell us what is going to happen in the general case. However, the most general case may need a separate careful investigation, which may prove that the outcome of the F-term uplifting is the same or better than the simplified case.

\section{Type IIA Compactification on $T^{6} / Z_{3}^{2}$ Orientifold}

The field content:

Since $h^{2,1}\left(T^{6} / Z_{3}^{2}\right)=0$, it has no complex structure modulus field. But it has the following Kähler moduli fields: it has 3 Kähler moduli $\left\{t_{i}, i=1,2,3\right\}$, each associated with one torus $T^{2}$. Also, it has 9 Kähler moduli $\left\{t_{B_{A}}, A=1,2, \cdots 9\right\}$ associated with 9 singularities of the orbifold (blow-up modes). Further, the dilaton $e^{-\phi}$ and its axionic partner $\xi$ are coming from the complex field $N_{0}$.

In short, this model has 12 complex fields (Kähler moduli) $\left\{t_{i}, t_{B_{A}} ; i=1,2,3, A=\right.$ $1,2, \cdots 9\}$, and an additional complex scalar, $N_{0}$, coming from the universal hypermultiplet after the orientifolding.

The fluxes:

Since there are no complex structure moduli, we do not have any flux which belongs to $H^{2,1}$. The only surviving part of the $H$ flux comes from $H^{3,0}$. Therefore, as far as the $H$ flux is concerned, we have only one flux which we denote it by $p$ (which is a constant). We also have RR-fluxes which come from $F_{0}, F_{2}, F_{4}$, and $F_{6}$ RR-forms. Let us parameterize 
the fluxes (after integration of $p$-forms on $p$-cycles) by:

$$
\begin{gathered}
F_{0} \Longleftrightarrow m_{0} \\
F_{2} \Longleftrightarrow\left\{m_{i}, n_{A} ; i=1,2,3, A=1,2 \cdots 9\right\} \\
F_{4} \Longleftrightarrow\left\{e_{i}, f_{A} ; i=1,2,3, A=1,2 \cdots 9\right\} \\
F_{6} \Longleftrightarrow e_{0}
\end{gathered}
$$

In short, $\left\{p, m_{0}, e_{0}, m_{i}, e_{i}, n_{A}, f_{A} ; i=1,2,3, A=1,2 \cdots 9\right\}$ parameterize all fluxes in the most general case for this orbifold.

The Kähler potential:

The components of intersection form of this orientifold are: $\kappa_{i j k}=\kappa\left|\epsilon_{i j k}\right|, \kappa_{A A A}=\beta$, and all other components vanish. The Kähler potential includes two parts: The part which depend on the Kähler moduli:

$$
K^{K}=-\log \left(-\frac{4}{3}\left(6 \kappa v_{1} v_{2} v_{3}+\beta \sum_{A=1}^{9} v_{b A}^{3}\right)\right)
$$

in which we have rewritten each complex field in terms of its real and imaginary parts: $t_{i}=b_{i}+i v_{i}, t_{B_{A}}=b_{B_{A}}+i v_{B_{A}}$, and the part which depends on $H$ flux:

$$
K^{Q}=4 D=\frac{e^{4 \phi}}{\operatorname{vol}^{2}}, \quad \operatorname{vol}=\kappa v_{1} v_{2} v_{3}
$$

The total Kähler potential is $K=K^{K}+K^{Q}$.

The superpotential:

The total superpotential is given by $W=W^{Q}+W^{K}$, where by $W^{Q}=-2 p N_{0}$ and

$$
W^{K}=e_{0}+e_{i} t_{i}+f_{A} t_{b A}+\frac{1}{2} \kappa\left|\epsilon_{i j k}\right| m_{i} t_{j} t_{k}+\frac{1}{2} \beta \sum_{A=1}^{9} n_{A} t_{B_{A}}^{2}-m_{0} \kappa t_{1} t_{2} t_{3}-\frac{m_{0}}{6} \beta \sum_{A=1}^{9} t_{b A}^{3}
$$

The supersymmetric solutions:

The supersymmetric solutions are given by (we denote the solutions by a star in order to keep things clear)

$$
\begin{gathered}
t_{i}^{*}=\frac{m_{i}}{m_{0}}+\frac{i}{\left|\hat{e}_{i}\right|} \sqrt{\frac{-5 \hat{e}_{1} \hat{e}_{2} \hat{e}_{3}}{3 m_{0} \kappa}}, \quad \text { where } \hat{e}_{i}=e_{i}+\frac{\kappa\left|\epsilon_{i j k}\right| m_{j} m_{k}}{2 m_{0}} \\
t_{B_{A}}^{*}=\frac{n_{A}}{m_{0}}-i \sqrt{\frac{-10 \hat{f}_{A}}{3 \beta m_{0}}}, \text { where } \hat{f}_{A}=f_{A}+\frac{\beta n_{A}^{2}}{2 m_{0}}
\end{gathered}
$$


and for the dilaton, we have

$$
e^{-\phi^{*}}=-\frac{4 \sqrt{3}}{15} \frac{m_{0}}{p}\left[\frac{10}{\left|m_{0}\right|} \sqrt{\frac{-5 \hat{e}_{1} \hat{e}_{2} \hat{e}_{3}}{3 m_{0} \kappa}}+\beta \sum_{A=1}^{9}\left(\frac{-10 \hat{f}_{A}}{3 \beta m_{0}}\right)^{3 / 2}\right]^{1 / 2},
$$

and finally for the axion $\xi$, we have

$$
\xi^{*}=\frac{1}{p}\left(e_{0}+\frac{e_{i} m_{i}+f_{A} n_{A}}{m_{0}}+\frac{6 \kappa m_{1} m_{2} m_{3}+\beta \sum_{A} n_{A}^{3}}{3 m_{0}^{2}}\right) \text {. }
$$

We make two simplifications:

1. We choose fluxes $F_{2}=0$, so that $m_{i}=n_{A}=0$. This leads to $\hat{e}^{i}=e^{i}$ and $\hat{f}_{A}=f_{A}$ and

$$
t_{i}^{*} \Rightarrow \frac{i}{\left|e_{i}\right|} \sqrt{\frac{-5 e_{1} e_{2} e_{3}}{3 m_{0} \kappa}}, \quad t_{B A}^{*} \Rightarrow-i \sqrt{\frac{-10 f_{A}}{3 \beta m_{0}}} .
$$

For the dilaton we have

$$
e^{-\phi^{*}} \Rightarrow-\frac{4 \sqrt{3}}{15} \frac{m_{0}}{p}\left[\frac{10}{\left|m_{0}\right|} \sqrt{\frac{-5 e_{1} e_{2} e_{3}}{3 m_{0} \kappa}}+\beta \sum_{A=1}^{9}\left(\frac{-10 f_{A}}{3 \beta m_{0}}\right)^{3 / 2}\right]^{1 / 2},
$$

and for the axion $\xi$ we have

$$
\xi^{*} \Rightarrow \frac{1}{p}\left(e_{0}\right)
$$

2. We assume that fluxes on non-blow-up modes dominate the string coupling, $\left|e_{i}\right| \gg\left|f_{A}\right| \gg\left|m_{0}\right|$. This leads to an approximate equation for the stabilized dilaton,

$$
e^{-\phi^{*}} \Rightarrow-\frac{4 \sqrt{3}}{15} \frac{m_{0}}{p}\left[\frac{10}{\left|m_{0}\right|} \sqrt{\frac{-5 e_{1} e_{2} e_{3}}{3 m_{0} \kappa}}\right]^{1 / 2} .
$$

\section{F-term uplifting of the simplified model}

The total potential is given by

$$
V_{\text {total }} \approx V_{I I A}+e^{K_{I I A}} V_{0}+\ldots
$$

where $V_{0}$ is the constant value of the potential from the $B$ sector. The uplifting term is given by the following expression:

$$
V_{u p} \approx e^{K_{I I A}} V_{0}, \quad e^{K_{I I A}}=e^{K^{K}+K^{Q}}=\frac{4 D}{\mathrm{vol}} .
$$

If the $B$ sector is given by the quantum corrected O'Raifeartaigh model [10], [11], we have $W_{O^{\prime}}=-\mu^{2} X$ and $K_{O^{\prime}}=X \bar{X}-\frac{(X \bar{X})^{2}}{\Lambda^{2}}$, so that at the minimum $e^{K_{O^{\prime}}} \approx 1$ and $V_{O^{\prime}}=V_{0} \approx\left|\frac{\partial W}{\partial X}\right|^{2}=\mu^{4}$. 
It is easy to study the uplifting in the simplified model since the potential depends only on 2 variables. As shown in [2],

$$
\frac{1}{\lambda} V_{I I A}=\left[\frac{1}{2} g^{4} r^{6}-\sqrt{2} g^{3}+\frac{1}{4} \frac{g^{2}}{r^{6}}+\frac{3}{2} \frac{g^{4}}{r^{2}}\right] .
$$

Here $v_{i}=\frac{v}{\left|e_{i}\right|}$, vol $=\frac{v^{3}}{\mathrm{E}}, E=\frac{\left|e_{1} e_{2} e_{3}\right|}{\kappa}, e^{D}=|p| \sqrt{\left|m_{0}\right| / E} g, v=\sqrt{\left|m_{0}\right| / E} r^{2}, \lambda=$ $p^{4}\left|m_{0}\right|^{5 / 2} E^{-3 / 2}$. The uplifting potential is positive and in these notation is given by

$$
V_{u p} \approx e^{K_{I I A}} V_{0} \sim \frac{g^{4}}{r^{6}}
$$

To see if this makes the negative cosmological constant positive we would prefer to deal with the potential depending on only one variable like in the KKLT scenario where one can easily see that the AdS minimum in $\sigma$ becomes a dS minimum meta-stable vacuum with the barrier separating the minimum from the Minkowski vacuum at infinite $\sigma$. Here $\sigma$ is related to the total volume of the compactified space. To proceed along these lines in type IIA model, we would need to fix the dilaton by a separate mechanism, make it much heavier than the volume modulus, and integrate it out, as in the IIB case. However, the quick glance on the mass matrix in type IIA theory presented in eq. (3.39) in [2] shows that no such situation can be easily found in the model [2], since the dilaton and the total volume are fixed by the same combination of fluxes. In contrast, in type IIB the dilaton is fixed by the electric and magnetic 3-form fluxes, whereas the volume is fixed by the gaugino condensates and/or Euclidean 3-brane instantons. Therefore the mass scales can be made very different. It has been shown in 12 that in the presence of the complex structure fields, such an hierarchy of mass scales can be achieved, and the uplifting can be viewed as if it takes place in the KKLT model with a single volume modulus.

Thus in the present situation we have to deal with the uplifting of the minimum by using the explicit expression for the potential for the 2-moduli problem,

$$
\frac{1}{\lambda} V_{\text {total }} \approx\left[\frac{1}{2} g^{4} r^{6}-\sqrt{2} g^{3}+\frac{1}{4} \frac{g^{2}}{r^{6}}+\frac{3}{2} \frac{g^{4}}{r^{2}}+c \frac{g^{4}}{r^{6}}\right] .
$$

Here the last term in the potential is the uplifting term with $c=\frac{V_{0}}{\lambda}>0$. First, we were using Mathematica to plot the potential as a function of 2 variables. At $c=0$ it has a clear minimum with the negative potential in the minimum. By gradually increasing the uplifting coefficient $c$, we expected to achieve the uplifting to a dS minimum. However, this was not happening: either there was a minimum with negative cosmological constant, or no minimum whatsoever. This particular behavior can be easily explained by calculating $6 g \partial_{g}-r \partial_{r}$ acting on the potential, following [2]. One finds that at the minimum

$$
\left(6 g \partial_{g}-r \partial_{r}\right) V_{t o t a l}=18 V_{t o t a l}+12 \lambda\left[\frac{g^{4}}{r^{2}}+c \frac{g^{4}}{r^{6}}\right]=0
$$


Therefore, despite our attempts to uplift the potential, its value at the minimum of the potential remains negative, ${ }^{1}$ and there are no minima for the positive values of the potential:

$$
V_{\text {total }}^{\min }=-\frac{2}{3}\left[\frac{g^{2}}{r^{6}}+c \frac{g^{4}}{r^{6}}\right]<0 .
$$

\section{Discussion}

One of the most interesting results of Ref. [2] was a discovery of an infinitely large number of stable AdS vacua in the type IIA massive string theory where the integer $N$ characterizing these vacua is unbounded. It has been argued in the literature and explained in the recent review [3] that the total number of vacua in this model is finite as a result of the physically motivated cut-off of the large volumes of compactification. But the main question remains whether any of these negative $\mathrm{CC}$ vacua could be uplifted and become the metastable $\mathrm{dS}$ vacua with positive $\mathrm{CC}$.

As we have shown, in the simplified version of the massive type IIA model [2] the Fterm uplifting mechanism does not work. One may try to improve this situation by relaxing our first assumption, i.e. by considering non-vanishing axions. However, since the Kähler potential does not depend on axions, it is unlikely that the "negative CC" conclusion will change. One may also try to relax our second assumption, and have the blow-up modes to change the dynamics of the stabilization significantly. Here it is easy to get into a conflict with the supergravity approximation, but it is not apriory clear that this is impossible. This possibility requires a separate investigation. Finally, one may consider a more general class of models based on the type IIA string theory and see whether the F-term uplifting can be implemented in any of these models. However, our results demonstrate that it may be a challenging task to uplift any of the infinite number of the AdS vacua found in [2] and make them phenomenologically acceptable. Additional studies of these issues may affect our understanding of the structure of the landscape and the criteria which should be applied towards the counting of quasi-realistic vacua.

\section{Acknowledgments}

It is a pleasure to thank S. Kachru, A. Linde, M. Peskin, S. Shenker and L. Susskind for stimulating discussions. This work was supported by NSF grant PHY-0244728. MS is also supported by the US Department of Energy under contract number DE-AC02-76SF00515.

\section{References}

[1] J. P. Derendinger, C. Kounnas, P. M. Petropoulos and F. Zwirner, "Superpotentials in IIA compactifications with general fluxes," Nucl. Phys. B 715, 211 (2005) [arXiv:hep-th/0411276]; G. Villadoro and F. Zwirner, "N = 1 effective potential from dual type-IIA D6/O6 orientifolds with general fluxes," JHEP 0506, 047 (2005)

\footnotetext{
${ }^{1}$ It was suggested to us by M. Peskin that one can try to use an uplifting term with the constant $c$ replaced by an exponential dependence on the coupling of the form $e^{-b(r) g}$. We leave this possibility for future investigations.
} 
[arXiv:hep-th/0503169]; J. P. Derendinger, C. Kounnas, P. M. Petropoulos and F. Zwirner, "Fluxes and gaugings: N =1 effective superpotentials," Fortsch. Phys. 53, 926 (2005) [arXiv:hep-th/0503229].

[2] O. DeWolfe, A. Giryavets, S. Kachru and W. Taylor, "Type IIA moduli stabilization," JHEP 0507, 066 (2005) [arXiv:hep-th/0505160].

[3] M. R. Douglas and S. Kachru, "Flux compactification," arXiv:hep-th/0610102.

[4] T. W. Grimm and J. Louis, "The effective action of type IIA Calabi-Yau orientifolds," Nucl. Phys. B 718, 153 (2005) [arXiv:hep-th/0412277].

[5] B. S. Acharya, F. Benini and R. Valandro, "Fixing moduli in exact type IIA flux vacua," arXiv:hep-th/0607223; T. Banks and K. van den Broek, "Massive IIA flux compactifications and U-dualities," arXiv:hep-th/0611185.

[6] S. Kachru, R. Kallosh, A. Linde and S. P. Trivedi, "De Sitter vacua in string theory," Phys. Rev. D 68, 046005 (2003) hep-th/0301240.

[7] V. Balasubramanian and P. Berglund, "Stringy corrections to Kaehler potentials, SUSY breaking, and the cosmological constant problem," JHEP 0411, 085 (2004) [arXiv:hep-th/0408054]; A. Westphal, "Eternal inflation with alpha' corrections," JCAP 0511, 003 (2005) [arXiv:hep-th/0507079]; A. Westphal, "de Sitter String Vacua from Kahler Uplifting," arXiv:hep-th/0611332.

[8] M. Gomez-Reino and C. A. Scrucca, "Locally stable non-supersymmetric Minkowski vacua in supergravity," JHEP 0605, 015 (2006) [arXiv:hep-th/0602246]. O. Lebedev, H. P. Nilles and M. Ratz, "de Sitter vacua from matter superpotentials," Phys. Lett. B 636, 126 (2006) [arXiv:hep-th/0603047]. F. Brummer, A. Hebecker and M. Trapletti, "SUSY breaking mediation by throat fields," Nucl. Phys. B 755, 186 (2006) [arXiv:hep-th/0605232].

[9] E. Dudas, C. Papineau and S. Pokorski, "Moduli stabilization and uplifting with dynamically generated F-terms," arXiv:hep-th/0610297; H. Abe, T. Higaki, T. Kobayashi and Y. Omura, "Moduli stabilization, F-term uplifting and soft supersymmetry breaking terms," arXiv:hep-th/0611024.

[10] R. Kallosh and A. Linde, "O'KKLT," arXiv:hep-th/0611183.

[11] L. O'Raifeartaigh, "Spontaneous Symmetry Breaking For Chiral Scalar Superfields," Nucl. Phys. B 96, 331 (1975).

[12] K. Choi, A. Falkowski, H. P. Nilles, M. Olechowski and S. Pokorski, "Stability of flux compactifications and the pattern of supersymmetry breaking," JHEP 0411, 076 (2004) [arXiv:hep-th/0411066]. 